\title{
[gw22-e0082] HISTOLOGICAL OBSERVATION OF UROKINASE AND GLYCOPROTEIN IIB/IIIA-TARGETED MICROBUBBLES PREPARED BY DIRECT CONJUGATION METHOD TO DISSOLVE THE THROMBI IN VIVO
}

Mu Yuming, Guan Lina, Wang Chunmei Department Of Echocardiography, Center Of Medical Ultrasound, The First Affiliated Hospital Of Xinjiang Medical University, Xinjiang, China

10.1136/heartjnl-2011-300867.141

Objective To observe the histological change of thrombi in vivo dissolved by urokinase and glycoprotein IIb/IIIa-targeted microbubbles combined with ultrasound in order to study the thrombolysis mechanism.

Methods A total of $42 \mathrm{New}$ Zealand white rabbits with platelet-rich thrombi in the femoral artery were randomised into seven treatment groups $(n=6)$ : (1) ultrasound alone (US); (2) ultrasound plus non-targeted microbubbles (US+M); (3) urokinase alone (UK); (4) ultrasound, non-targeted microbubble and urokinase (US+M+UK); (5) ultrasound plus platelet-targeted microbubble (US+R); 6) platelet-targeted microbubble plus urokinase $(\mathrm{R}+\mathrm{UK})$; and $(7)$ ultrasound, platelet-targeted microbubble and urokinase (US+R+UK). Urokinase and RGDS were in conjunction with the surface of commercial microbubbles (SonoVue) by direct conjugation method. US was simultaneously applied transcutaneously over the thrombus up to $30 \mathrm{~min}$. The thrombolytic effect was based on ultrasound, blood flow and histological observations at $120 \mathrm{~min}$ post treatment.

Results US+R+UK group resulted in the complete recanalisation $(p<0.001)$. HE staining showed that thrombus is completely dissolved. Scanning electron microscope examination showed thrombosis of the fiber network structure damage. Transmission electron microscopy revealed that thrombus was dissolved into high electron density particles. Expression of vWF and TF staining was negative.

Conclusions Damage of thrombus fibrin network structure, dissolution fibrin and prevention of the thrombus reformation are the main histological changes of thrombi in vivo dissolved by urokinase and glycoprotein IIb/IIIa-targeted microbubbles combined with ultrasound. 